\title{
Investigating the Idiotop Paratop Interaction in the Artificial Immune Networks
}

\author{
Hossam Meshref, Member IEEE \\ Computer Science Department \\ College of Computers and Information Technology \\ Taif University, Taif, Saudi Arabia
}

\begin{abstract}
The artificial immune system is a new computational intelligence technique that has been investigated for the past decade. By reviewing the literature, two observations were found that could affect the network learning process. First, most researchers do not focus on Paratop-Epitop and ParatopIdiotop interactions within the network. Second, most researchers depict the interaction within the network with all the network components present from the beginning until the end of the learning process. In this research, efforts were devoted to deal with the aforementioned observations. The findings were able to differentiate between interactions in a node within a network, and total interactions in the network. A small simulation problem was used to show the effect of choosing a steady number of antibodies during network interactions. Results showed that a considerable number of interactions could be saved during network learning, which will lead to faster convergence. In conclusion, it is believed that the designed model is ready to be used in many applications. Therefore, it is recommend the use of our model in different applications such as controlling robots in hazardous rescue environment to save human lives.
\end{abstract}

Keywords-Artificial Immune Systems; Idiotopic Networks; Paratop-Epitop; Paratop-Idiotop

\section{INTRODUCTION}

The artificial immune system is a young field and many researchers are trying to explore its boundaries to utilize it in different applications. However, it is not new that research efforts were invested to understand different biological processes through modeling. For example, artificial neural networks have been investigated thoroughly in the past three decades and a plethora of applications has been developed based on its modeling.

The artificial immune system is modeled after the biological immune system, which has many useful features. For example, it adapts to changes in an environment, it has hierarchical organization, and its control is distributed. The biological immune system consists mainly of Lymphocytes that have two major types: T-cells and B-cells. B-cells are responsible for humoral immunity that secretes antibodies. On the other hand, T-cells are responsible for cell mediated immunity. Each B-Cell has a unique structure that produces suitable antibodies in response to invaders of the system. That type of response is called innate immunity and eventually results in antibody-antigen relations to be stored in case the host encounters the same invader again. In that case, the immune response in expected to be faster given that the network have seen it before, i.e. learned how to deal with it [1].

Early researchers discovered that the immune system has Idiotopic networks that use stimulation and suppression among its elements to achieve immunity against antigens (Ags). The part of an antigen that could be recognized by antibodies is called epitope (Ep). It worth mentioning that a regular Ag may carry more than one epitope, and the result of this stimulation is that the B-cells start to produce Abs. On the other hand, the part of the antibody that can recognize epitopes of antigens is called paratope (P). Surprisingly, part of an antibody, called idiotope (Id), could be regarded as antigen by other antibodies' in the idiotopic network [2].

The basic idea of Idiotopic Network Hypothesis is that the Lymphocytes in this network communicate among each other mutually to gain immunity. Ag stimulates their matching paratops as key to the lock relation. On the other hand, the idiotop Id1 of Ab1 will stimulate the paratop P2 of B cell 2, which has Ab2 attached to it. Alternatively, from B cell \#2 point of view, we find that Id 1 acts as an Ag. Consequently, Ab1 with B cell \#1 will be suppressed by Ab2. From another prospective we will find that $\mathrm{Ab} 2$ stimulate $\mathrm{Ab} 1$ through Id2 [3]. The whole network members will mutually stimulate and suppress each other with a closed loop chain that acts as a self non-self recognizer, and eventually the suitable response to the Ag will evolve, see figure 1: a, b.

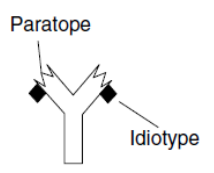

(A)

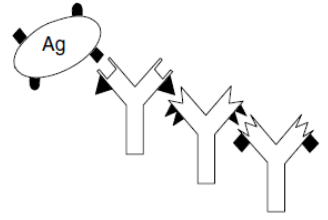

(B)
Fig. 1. (a) Antibody structure (b) Stimulation and supression in Idiotopic networks

As a brief of the structure of this paper, section two give an idea about the background research that was found in the literature. From that section, moving to section three, an in depth investigation of the interactions within a node is presented. Finally, in section four, research results are discussed along with some research directions for interested researchers, who may want to pursue their research using the presented findings. 


\section{BACKGROUD}

In this research the interaction within the artificial immune network is going to be investigated to understand its dynamics. The main focus will be on modeling the interaction between paratops and idiotops as well as the interactions between paratops and epitops in the network. It is believed that these interactions govern the behavior of the network and affects its learning ability. It is the interest of this proposed research to investigate the nature of that interaction and investigate how it affects the artificial immune network performance.

Most of the research found in the literature models the interaction within the network based on an assumption that the network has $n$ components from the beginning of the simulation until the end. Take applications on the field of robotics for example. Dasgupta and Nino surveyed robotics research inspired by Artificial Immune Networks. Among research done on autonomous robots, few robots utilized a mechanism called Immunoid to collect various amount of garbage from a constantly changing environment [4]. Singh and Thayer used a computer simulation of mobile robots trying to clear mines from a minefield using a response pattern based on the immune system [5]. Lau and Ko used a general suppression control framework that is based on suppression mechanism of immune cells. Their target was to design a robot search and rescue system [6,7]. However, in the aforementioned research, there was no deep investigation on the nature of the P-Ep and the P-I interactions in the network.

On the other hand, there were many applications that benefit from a few immune system features like self non-self discrimination, specificity, and memory. Several research efforts got advantage of such type of networks in the field of autonomous robots, e.g. [8,9]. The main idea behind their research was having a network that adapts itself by adjusting the concentrations of its nodes, Abs in each B-Cell, in a way that fulfills the overall objective function of the network. Each node in that network was a robot that has certain set of behaviors, and collectively the network adapts robots behaviors as they collaborate to achieve an overall common goal. However, there was no clear distinction between the P-Ep and the P-I interactions in the network. There was other applied research in the literature that used featured algorithms of the immune system such as the negative selection algorithm as well as clonal selection algorithm $[10,11]$. In addition, there was research that utilized the pattern recognition ability of the immune system $[12,13,14]$ and many other features.

In the surveyed research efforts, all robots' behaviors (Abs) were assumed to exist in the networks dynamics since $t_{1}$ of the simulation process. However, in this research, this assumption is seen differently. It is believed that a node, B-Cell, in the network starts at $t_{1}$ with an $\mathrm{Ag}$ and one corresponding antibody Ab1. Next, at $t_{2}$, as another antibody Ab2 is added to the network, the Idiotop of Ab1 recognizes Ab2 as an Ag as well. This means $\mathrm{Ab} 2$ receives stimulation from the $\mathrm{Ag}$ as well as the Idiotop of Ab1. On the other hand, Ab2 suppresses the Ag as well as the Idiotop of Ab1.

The process is repeated as a new antibody Ab3 is added to the network at $t_{3}$. Eventually, as the size of the network increases, the stimulation and suppression level should also increase in an augmenting manner. In our proposed research, we need to investigate that augmentation effect in depth to see the difference that it makes on the network performance. We need to find out whether or not that augmentation effect will improve the learning process. Unpacking the network structure concept will help us, as well as other researchers, to reconfigure artificial immune networks that have a more robust design. That design is expected to be capable of handling many challenges in the computational intelligence field.

To summarize, there are two assumptions investigated in this proposed research. First, we assume that there is a differentiation between P-Ep and P-I interactions in the network. Second, we assume that the interaction within the network is Augmentative and it may affect training. It is the interest of our research to investigate those hypotheses to be able to build a more robust network. That network, in turn, should produce results that are more accurate. In the following section, the design part is going to be discussed to show how we are going to conduct the proposed research.

\section{INVESTIGATING INTERACTIONS WITHIN THE IDIOTOPIC IMMUNE NETWORKS}

\section{A. Building up the Augmentation Concept During Node Interactions}

The main idea in our proposed research is based upon assuming that the node interactions increase in an augmentative manner. Table 1 shows the corresponding node interactions at different simulation times $t_{1}$ to $t_{n}$. Node structure including stimulation and suppression links, from times $t_{1}$ to $t_{3}$, are shown in figures 2-4. In this research, this interaction build up process is called the Augmentation Effect.

TABLE1. NODE AUGMENTATIVE INTERCTIONS

\begin{tabular}{|c|c|c|}
\hline $\begin{array}{l}\text { Simulation } \\
\text { Time }\end{array}$ & $\begin{array}{l}\text { Network } \\
\text { Components }\end{array}$ & $\begin{array}{l}\text { Type of } \\
\text { Interaction }\end{array}$ \\
\hline$t_{1}$ & $\mathrm{Ab} 1 \& \mathrm{Ag}$ & P-Ep \\
\hline \multirow{3}{*}{$t_{2}$} & $\mathrm{Ab} 1 \& \mathrm{Ag}$ & P-Ep \\
\hline & $A b 1 \& A b 2$ & P-I \\
\hline & $\mathrm{Ab} 2 \& \mathrm{Ag}$ & P-Ep \\
\hline \multirow{6}{*}{$t_{3}$} & $\mathrm{Ab} 1 \& \mathrm{Ag}$ & P-Ep \\
\hline & $A b 1 \& A b 2$ & P-I \\
\hline & $A b 1 \& A b 3$ & P-I \\
\hline & $\mathrm{Ab} 2 \& \mathrm{Ag}$ & P-Ep \\
\hline & $A b 2 \& A b 3$ & P-I \\
\hline & Ab3 \& Ag & P-Ep \\
\hline
\end{tabular}

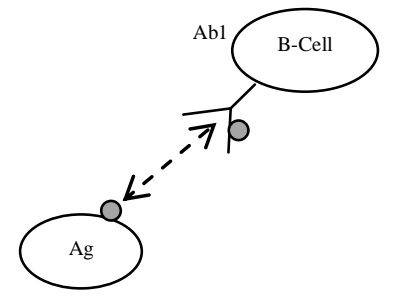

Fig. 2. Node interactions at $t_{1}$ 


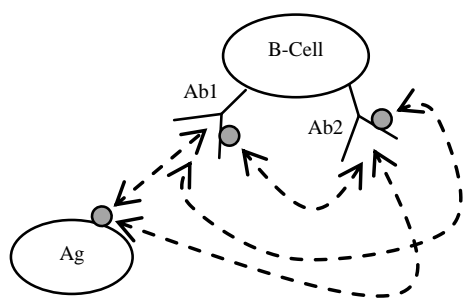

Fig. 3. Node interactions at $t_{2}$

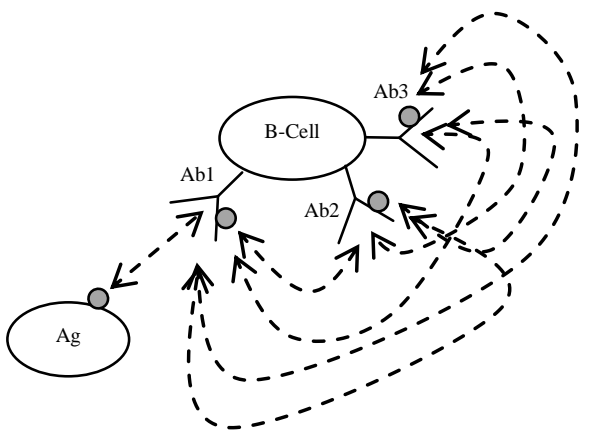

Fig. 4. Node interactions at $t_{3}$

\section{B. Concentrations within the Idiotopic Network}

Most researchers agreed that interactions within the Idiotopic immune network could be governed by equation 1 :

$\frac{d c_{i}(t)}{d t}=\left(\sum_{j=1}^{N} m_{j, i} c_{j}-\sum_{k=1}^{N} m_{i, k} c_{k}+m_{i}-k_{i}\right) c_{i}(t)$

The first term, from the left, represents the total stimulation between different B-cells in the idiotopic network. The second term represents the total suppression. The third term represents the $\mathrm{Ab}$ stimulation received by an $\mathrm{Ag}$ based on the affinity between them. As for the fourth term, $\mathrm{k}$, it represents the mortality of an $\mathrm{Ab}[15] . N$ represents the number of Abs, while $C$ represents the concentration of $\mathrm{Ab}$ within the network, and $m$ represents affinity.

To model concentrations for antigens and antibodies, theoretical biology should be involved. According to Boer, population growth could be described by a classical logistic equation [16]. Therefore, in this research, antibody concentration could be modeled mathematically using equations 2 and 3 :

$C_{A b}=\frac{C_{A b_{\circ}}+C_{A b_{\max }}}{C_{A b_{\circ}}+\left(C_{A b_{\text {max }}}-C_{A b_{\circ}}\right) e^{-r t}}$

$r=b-d$

, where $\mathrm{C}_{\mathrm{Ab}}$ 。 is the initial concentration of the $\mathrm{Ab}$ population, $\mathrm{C}_{\mathrm{Ab} \text { max }}$ is the carrying capacity of the population, and $r$ is the natural rate of increase represented as the difference between the birth rate, $b$, and the death rate $d$.
The Ag population mathematical representation is in general similar to the $\mathrm{Ab}$ abovementioned representation. However, in this research, the Ag is not assumed to proliferate. Unlike the $\mathrm{Ab}$ concentration equation, it is expected that as $t \rightarrow \infty$ the Ag concentration will approach Zero $\left(C_{A g} \rightarrow 0\right)$. The $\mathrm{Ag}$ concentration could be modeled mathematically using equation 4 :

$C_{A g}(t)=C_{A g_{\max }} e^{-d t}$

All the aforementioned relations and algorithms are going to be deployed in the investigation of the relations within the Artificial Idiotopic Immune Network. In the following section, we are going to present our findings regarding the interactions within a node in the network as well as the interactions within the overall network as a whole.

\section{RESULTS AND DISCUSSION}

A. Investigating the overall number of interactions in a B-cell node within the Network

As mentioned earlier, according to most of the research in the surveyed literature ranging from 2005 to 2013, there was no differentiation between P-Ep and P-I interactions within the network. In addition, most of the literature depicts the interaction within the node with all the node components present from $t_{1}$.

Based on the augmentative interaction details shown in table 1, we find that the number of P-Ep interactions is going to be $n$, which is the number of antibodies in the network. On the other hand, we find that the number of P-I interactions is going to be $\left(\begin{array}{l}n \\ 2\end{array}\right)$ assuming that there is no repetition and that order does not matter, see figures 5 and 6 .

As illustrated in figure 7, by combining the number of P-Ep interactions, $I_{P-E p}$, and the number of P-I interactions, $I_{P-I}$ ,within the artificial idiotopic network on one graph we can arrive at the following observations:

1) The number of interactions, $I_{P-E p}$ as well as $I_{P-I}$, are equal at $n=3$.

2) In addition, for $n: 0 \rightarrow 3, I_{P-E p}$ interactions are greater than $I_{P-I}$ interactions.

3) Conversely, for $n>3, I_{P-I}$ interactions are greater than $I_{P-E p}$ interactions.

4) By investigating the total number of interactions, $I_{T}$, within the node, we find that:

$I_{T}(n)=n+\left(\begin{array}{l}n \\ 2\end{array}\right)$

$I_{T}(n)=n+\frac{n(n-1)}{2}$

$I_{T}(n)=\frac{n^{2}+n}{2}$ 


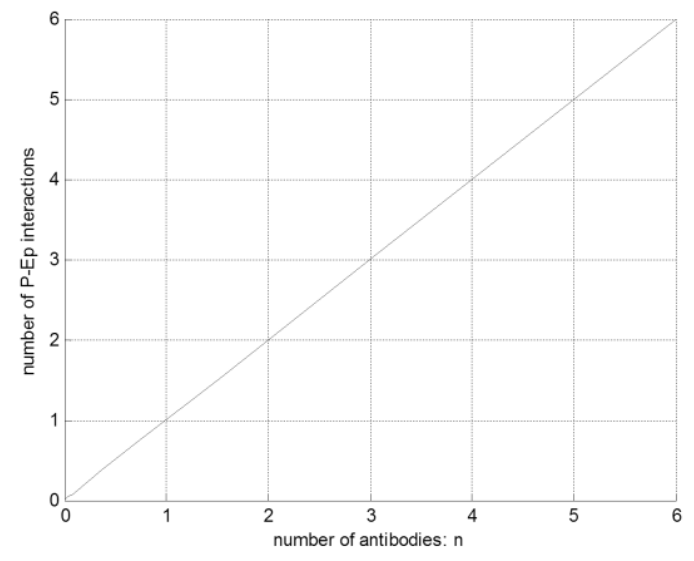

Fig. 5. The number of P-Ep interactions

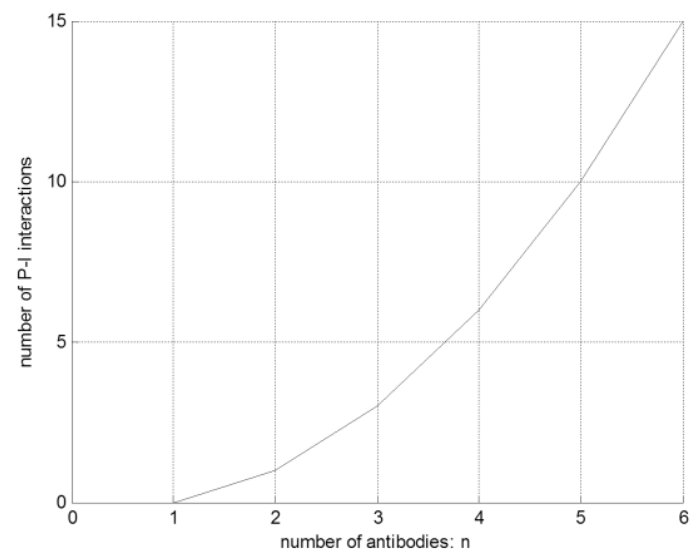

Fig. 6. The number of P-I interactions

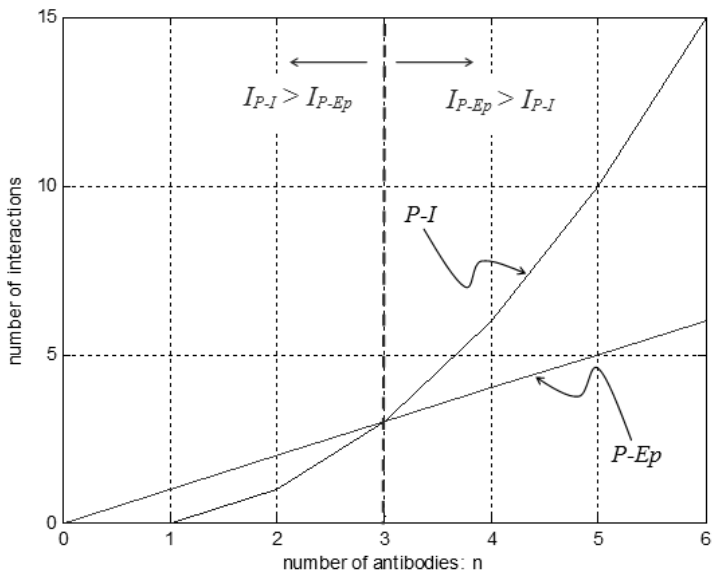

Fig. 7. Combined network interactions
In this research, it is believed that the P-I interaction continues to have the augmentation effect until the node is settled. In our design, it is expected that at a certain point, a number of antibodies $n^{*}$ will be enough to defeat the encountered antigen(s). At that time, there will be a steady pool of antibodies to select from based on highest concentrations. Later on during learning, as the learning process converges and the target is reached, memory cells are generated for future encounters of that antigen. That concept was investigated further to control the interactions within a node, and eventually deal with the augmentation effect.

It was found that it is not practical to limit the number of generated Abs during learning, because that will limit the chances of producing proper solution that counter the $\mathrm{Ag}$ effect. One possible approach in this research was to control the difference between the number of P-Ep interactions, $I_{P-E p}$, and the number of P-I interactions, $I_{P-I}$, within the artificial idiotopic network. By doing that, the number of interactions during learning could be controlled, and eventually that could reduce the augmentation effect. The difference in interactions $I_{d}$, could be analyzed using equation 6 :

$I_{d}(n)=n-\left(\begin{array}{l}n \\ 2\end{array}\right)$

$I_{d}(n)=\frac{n}{2}(3-n)$

To be more precise, we can still describe the difference in interactions for different time intervals using equation 7 :

$I_{d}(n)=\left\{\begin{aligned} \frac{n}{2}(3-n), & n \leq 3 \\ -\frac{n}{2}(3-n), & n>3\end{aligned}\right.$

, which could be modeled using the following equation:

$I_{d}(n)=\left\|\frac{n}{2}(3-n)\right\|$

It was found that the difference is steady until $t_{2}$ and then the network reaches a balance point at $n=3$, where both $I_{P-E p}$ and $I_{P-I}$ interactions are equal. For the difference in interactions increases in an augmentative manner, see figure 8.

As we focus on solving the augmentation problem to reach steady state interactions, it is believed that one possible solution is to argue that the $I_{d}(n)$ is not increasing. Therefore, the number of interactions is controlled. That goal could be achieved by keeping the number of antibodies constant in the network. In other words, the death and birth rates: $d$ and $r$ should be controlled to have a steady number of antibodies all the time, as in equation 9:

$d-r=n^{*}$ 


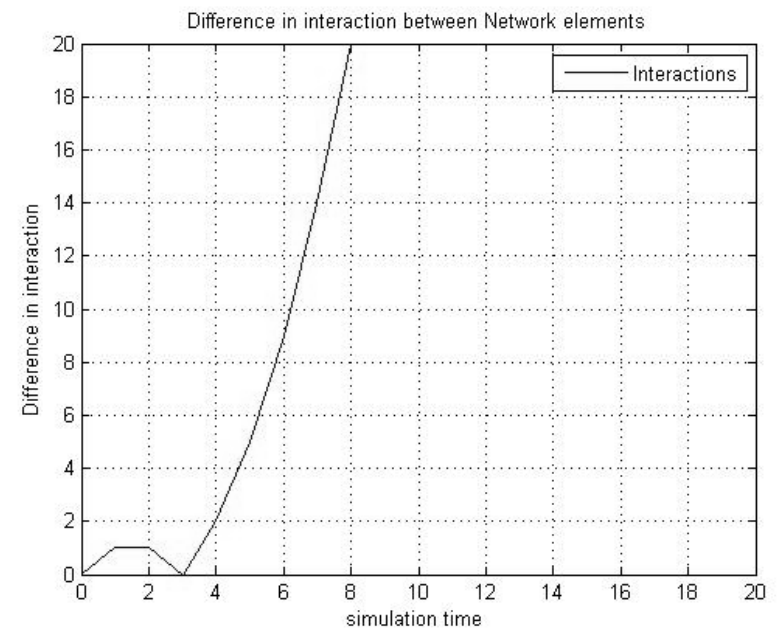

Fig. 8. Difference in interactions within a node

Based on the abovementioned assumption, we assum that as $n$ increases with values greater than 3 , the difference in interactions should increase until $n$ reaches a steady state value $n^{*}$. At that point, the node is presumably stable, see figure 9 (as an example, where $n^{*}=7$ ).

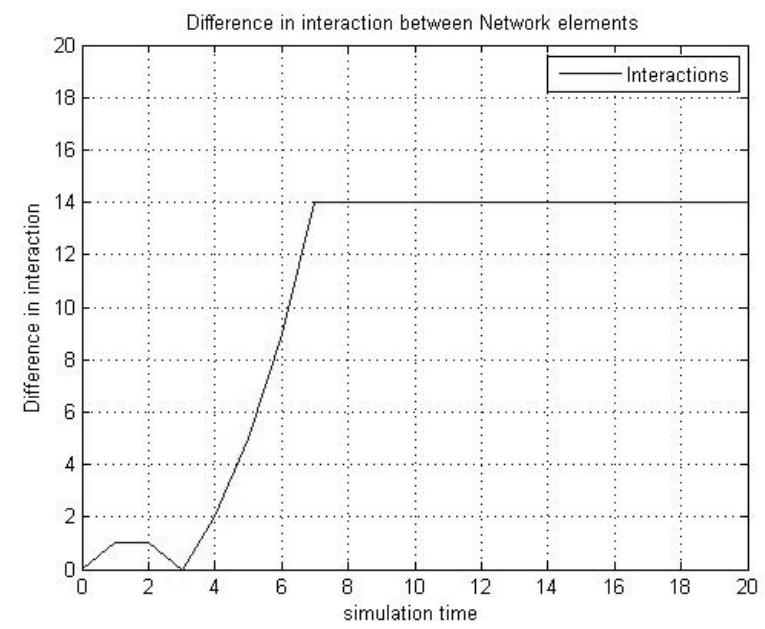

Fig. 9. Simplified P-Ep node interactions at $\mathrm{n}^{*}=7$

Based on figure 9, we could still describe the difference in interactions within a node for different time intervals using equation 10:

$I_{d}(n)=\left\{\begin{aligned} \frac{n}{2}(3-n), & n \leq 3 \\ -\frac{n}{2}(3-n), & n>3 \\ -\frac{n^{*}}{2}\left(3-n^{*}\right), & n \geq n^{*}\end{aligned}\right.$

By investigating the overall interactions within a node in the immune network, we reach the following findings:

1) Each node (B-Cell) has its own P-I \& P-Ep interactions, which are calculated using different equations.

2) Only within each node, we have augmentation effect. That counters our initial research hypothesis that we assumed at the beginning. However, if we consider other B-Cells in the overall network at the beginning of interactions, the number of $P-I \& P$-Ep interactions will be greater than the values mentioned in table 1. To understand how this is actually implemented, consider the case where the network has multiple B-Cells. Therefore, at $t_{1}$ we could have multiple Abs. For example, there would be $m A b s$, where $m$ is the number of $B$-Cells in the network, and assuming that each B-Cell, node, starts with One Ab in its interaction. At that point, there will be more than just one P-Ep interaction to start with at $t_{1}$.

\section{$B$. Investigating the overall number of interactions in the network}

Based on the aforementioned findings, we concluded that the total interactions within the node could be given by:

$I_{T}(n)=\frac{n(n+1)}{2}$

Where $n$ is the number of antibodies in a node. Therefore, the summation of all the antibody interactions in $m$ nodes in the network could be:

$$
\begin{aligned}
& I_{T_{n e t}}=\sum_{i=1}^{m} I_{T}\left(n_{i}\right) \\
& I_{T_{n e t}}=\frac{n_{1}\left(n_{1}+1\right)}{2}+\frac{n_{2}\left(n_{2}+1\right)}{2}+\cdots+\frac{n_{m}\left(n_{m}+1\right)}{2}
\end{aligned}
$$

A special case occurs when each B-Cell has the same number of Ab's (behaviors). In that case:

$$
\begin{aligned}
& I_{T_{n e t}}(n)=\frac{m * n(n+1)}{2} \\
& I_{d_{n e t}}(n)=\left\|\frac{m * n}{2}(3-n)\right\|
\end{aligned}
$$

\section{Testing the Findings on a Simple Robotics Simulation Problem}

In a general case, we should have $m$ robots (B-Cells) cooperating in an environment to achieve a certain goal. Each robot is assumed to have $n$ behaviors (Antibodies). It is recommended to have a smaller number of behaviors to be able to study the effect on learning. Therefore, in this simulation we choose a simple problem of a robot that has small number of behaviors. The reason behind that choice is to focus on the analysis of the number of interactions that could be saved during simulation. In this simulation problem, we have one robot. That robot has four behaviors, which are move west, move east, move north, and move south. Each behavior has its own concentration. The robot is attempting to reach a target area autonomously by trying to minimize the distance to target value.

Using the total interactions equation in this simulation, while choosing $n^{*}=2$, resulted in a total number of 3 interactions within the network. In this problem simulation, the selection of $n^{*}=2$ is based on choosing $b-d=2$, which means there were two selected behaviors with the highest concentrations from the pool of possible four behaviors. Choosing $n^{*}=2$, we saved $70 \%$ of the number of calculations during simulation. Figure 10 shows the change in behavior concentrations during simulation until the robot reached the desired target location. 
Change in concentration of Antibodies
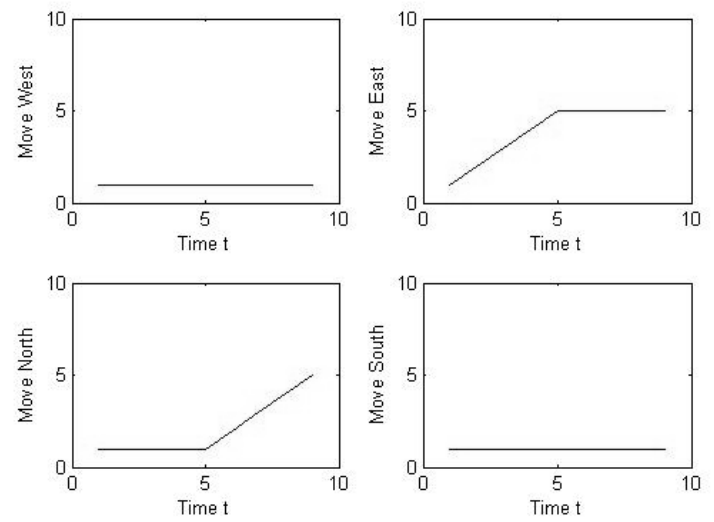

Fig. 10. Behavior concentrations within the IAIN

By running different simulations, it was found that as $n$ increases, the percentage of total number of interactions saved, $S(n)$, increases based on equation 14:

$S(n)=1-\frac{n^{*}\left(n^{*}+1\right)}{n(n+1)}$

, where for example, $n^{*}=2$, based on the run of previous simulation and $n \geq n^{*}$. The preceding findings proved that the choice of $n^{*}$ out of $n$ from the pool of possible behaviors affects learning simulation time, because a few calculation steps are saved. On the other hand, in another case, choosing two behaviors $\left(n^{*}=2\right)$ out of a pool of three behaviors could save $50 \%$ of the number of calculations. Figure 11 shows that the percentage of calculations saved increased, and as a result, we could have faster convergence as the learning speed increased.

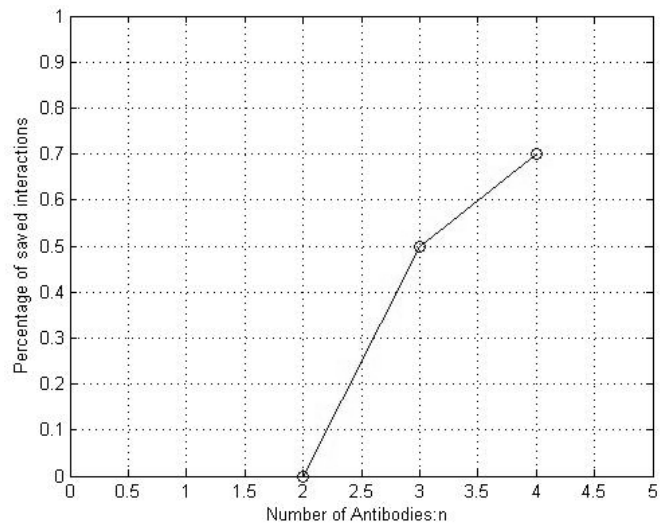

Fig. 11. Percentage of saved interactions

\section{CONCLUSIONS AND RECOMMENDATIONS}

Artificial Immune networks have been a reliable model for the past decade. In this research, a new trend was used based on unpacking the network ideology regarding the number of interactions within the network. On the other hand, the careful design scaffolding process that we implemented in analyzing the augmentation effect within a node resulted in several useful equations that could describe different types of node interactions, and eventually resulted in a more robust network.

The resulted interaction model suits different applications that deploy intelligent behavior. It is recommend to use the designed network model to build some applications in the in the robotics field, for example, where robots can be used in rescue operations. These types of applications can intervene in hazardous situations and save many human lives.

\section{REFERENCES}

[1] Ehud Lamm , Ron Unger, Biological Computation: Chapman and Hall/CRC Publishing, 2011.

[2] Leandro Nunes de Castro, Jonathan Timmis, Artificial Immune Systems: A New Computational Intelligence Approach: Springer, 2002.

[3] Hongwei Mo, Handbook of Research on Artificial Immune Systems and Natural Computing: Applying Complex Adaptive Technologies: Igi Global Publisher, 2009.

[4] Dipankar Dasgupta, Luis F. Niño, Immunological Computation: Theory and application. NewYork, NY : CRC Press, 2009.

[5] D. Dasgupta, "Advances in Artificial Immune Systems," IEEE Computational Intelligence Magazine, Vol. 1, No. 4, pp. 40-49, 2006.

[6] Singh, S. P. N. and S. M. Thayer, "Immunology directed methods for distributed robotics: A novel, immunity-based architecture for robust control \& coordination." The Proceedings of SPIE: Mobile Robots XVI, Vol. 4573, November 2001.

[7] Lau, H. Y. K. Lau, and A. Ko, "An immuno robotic system for humanitarian search and rescue." ICARIS'07 Proceedings of the 6th international conference on Artificial immune systems, pp.191-203, 2007.

[8] Meshref, H., "The Rod-Bearing Problem: A Cooperative Autonomous Robotics Application Based on Artificial Idiotopic Immune Networks," Systems, Man, and Cybernetics (SMC), 2013 IEEE International Conference on, vol., no., pp.1654,1659, 2013.

[9] Yang He; Liang Yiwen; Li Tao; Zhou Ting, "A model of Collaborative Artificial Immune System," Informatics in Control, Automation and Robotics (CAR), 2010 2nd International Asia Conference on , vol.3, no., pp.101,104, 6-7, 2010.

[10] Ying Tan; Guyue Mi; Yuanchun Zhu; Chao Deng, "Artificial immune system based methods for spam filtering," Circuits and Systems (ISCAS), 2013 IEEE International Symposium on , vol., no., pp.2484,2488, 19-23 May 2013.

[11] Purbasari, A.; Iping, S.S.; Santoso, O.S.; Mandala, R., "Designing Artificial Immune System Based on Clonal Selection: Using AgentBased Modeling Approach," Modelling Symposium (AMS), 2013 7th Asia , vol., no., pp.11,15, 2013.

[12] Nemmour, H.; Chibani, Y., "Off-line signature verification using artificial immune recognition system," Electronics, Computer and Computation (ICECCO), 2013 International Conference on , vol., no., pp.164,167, 7-9 Nov. 2013.

[13] Yu Xiao; Fu Dongmei; Yang Tao, "Application of artificial immune algorithm in image segmentation based on immune field," Intelligent Control and Automation (WCICA), 2012 10th World Congress on , vol., no., pp.4691,4695, 6-8 July 2012.

[14] Simon M. Garrett, "How Do We Evaluate Artificial Immune Systems?" Journal of Evolutionary Computation, 13(2), pp.145-178, 2005.

[15] Farmer, J. D., N. H. Packard and A. S. Perelson. The immune system, adaptation and machine learning. Physica, 22D, 187-204, 1986.

[16] Rob J. de Boer. Theoretical Biology \& Bioinformatics: Utrecht University Press, 2013. 